\title{
The physiology of thyroid function in pregnancy
}

\author{
JOHN FEELY \\ M.B., B.Sc., M.R.C.P.I. \\ Department of Pharmacology and Therapeutics, Ninewells Hospital and Medical School, \\ Dundee DDI 9SY, Scotland
}

\begin{abstract}
Summary
Pregnancy has a variety of effects on maternal thyroid function. Thyroid gland enlargement is common particularly in areas of relative iodine deficiency. The renal clearance of iodine is increased in pregnancy and together with an increased volume of iodine distribution, leads to a low plasma inorganic iodine and thus increases the thyroidal iodine clearance. However, the absolute iodine uptake and hormone production rate remain unchanged.
\end{abstract}

There is an increase in the serum thyroxine $\left(T_{4}\right)$ and triiodothyronine $\left(T_{3}\right)$ concentration largely due to an increase in thyroid hormone-binding proteins. Free thyroxine, and free $T_{3}$ remain unchanged in pregnancy as does the Free Thyroxine Index, which gives the single most accurate measure of thyroid function. The placenta secretes a number of thyroid stimulators including human chorionic gonadotrophin and possibly chorionic thyrotrophin and molar thyrotrophin whose physiological role is to date poorly understood.

The fetal thyroid develops independently, and although fetal $T_{4}$ concentration rises progressively to maternal by term, the $T_{3}$ concentration is markedly reduced owing to preferential formation of inactive reverse $T_{3}$.

\section{Introduction}

The observation that physiological changes in thyroid function occur in pregnancy is neither new nor is it surprising. Pregnancy goitre has been recognized for some 7000 years, and more recently a clearer understanding of its development has emerged. Four major changes (Fig. 1) occur in maternal thyroid physiology during pregnancy: (i) enlargement of the thyroid, (ii) alterations in iodine handling, (iii) an increase both in thyroid hormone binding proteins and thyroid hormones and, (iv) the production of placental thyroid stimulators. In addition, recent knowledge of the physiology of the fetal thyroid permits routine screening for hypothyroidism in the newborn.

Thyroid gland enlargement

The prevalence of goitre in pregnancy varies according to the area studied and the definition of goitre. Using a standardized procedure, Crooks et al. (1964) showed that $70 \%$ of 184 pregnant women in Aberdeen had both visible and palpable enlargement of the thyroid compared with $37 \%$ of non-pregnant age-matched controls. The distribution of goitre in pregnancy was not influenced by parity, maternal age, or stage of gestation. In a comparative study (Crooks et al., 1967) with an area of high iodine intake (Iceland), there was no evidence of thyroidal enlargement in pregnancy, and goitre was equally common $(20 \%)$ in both the control and pregnant groups. There is histological evidence of large follicles and abundant colloid, suggestive of active formation and secretion of thyroid hormones (Burrow, 1975). It has also been suggested (Tunbridge and Hall, 1975) that in addition to the possible stimulus of relative iodine deficiency, a proportion of the enlargement may also result from increased blood flow which is sometimes sufficient to produce a vascular bruit.

The modulator of thyroid enlargement in pregnancy is not fully clear. Thyroid stimulating hormone (TSH) levels remain within the normal range for most of pregnancy (Chan, Paraskevaides and Hale, 1975) although it is possible that there is an initial TSH stimulus in early pregnancy (Malkasian and Mayberry, 1970), sufficient to compensate for the increased requirements due to changes in hormone binding, which needs only to be temporary, as thyroxine requirements during pregnancy are unchanged (Dowling, Appleton and Nicoloff, 1967). It is also possible that human chorionic gonadotrophin (HCG; having a common alpha subunit with TSH) or human chorionic thyrotrophin (HCT) may be the goitrogenic stimulus. Alterations in iodine handling
Alterations in iodine handling occur early in
pregnancy. An increased renal clearance of iodine
which cannot be wholly explained by an increase in
glomerular filtration rate occurs throughout preg-
nancy and for up to 6 weeks post partum (Aboul-
Khair et al., 1964). This is associated with a fall in 


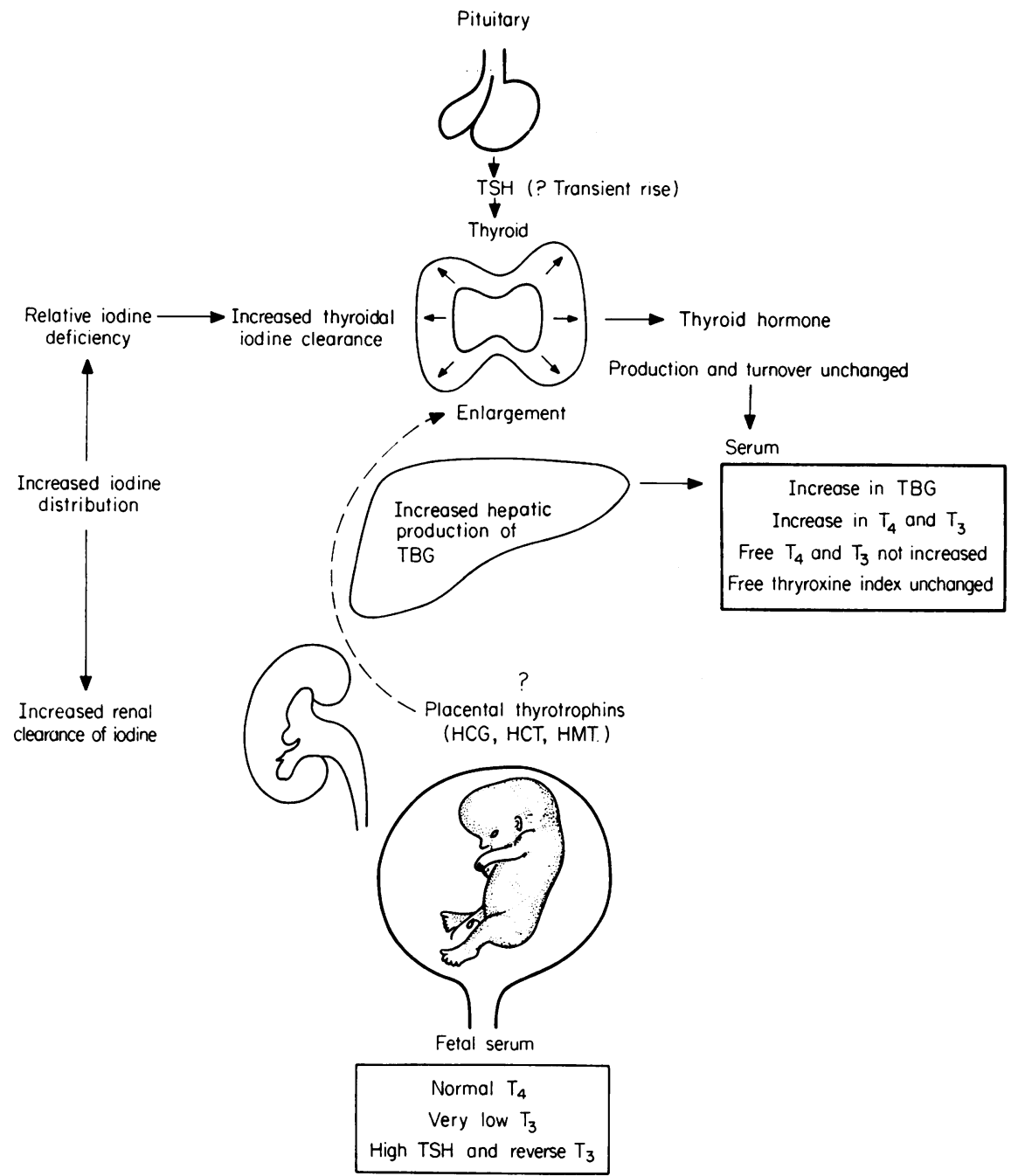

FIG. 1. Summary of the physiological changes in thyroid function during pregnancy. HCG, chorionic gonadotrophin; HCT, human chorionic thyrotrophin; HMT, human molar thyrotrophin; TBG, thyroxine-binding globulin; TSH, thyroid-stimulating hormone.

the plasma inorganic iodine concentration, in part due to haemodilution, increased iodine distribution volume, and maternal losses to fetus, and thus a compensatory increase in the thyroidal clearance of iodine. The absolute iodine uptake (an approximate index of thyroid hormone production) is, however, unchanged throughout pregnancy (Aboul-Khair et al., 1964). These studies were carried out in an area of relatively low iodine intake (Aberdeen) and may in part explain the high incidence of pregnancy goitre in this area in comparison to the lower incidence found in Iceland and in certain areas of the United States where the iodine intake is high. Although iodine supplements may correct many of these abnormalities, they are not however indicated in pregnancy, save in areas of severe iodine deficiency with a high incidence of endemic cretinism. In fact, excess iodine intake in the treatment of goitre, asthma or chronic cough has been associated with over $\mathbf{4 0 0}$ cases of neonatal goitre, including massive obstructive goitrous hypothyroidism and neonatal death (Galina, Avnet and Einhorn 1962).

\section{Thyroid hormones}

The frequent occurrence of goitre, and the apparent increase in thyroid hormone concentration 
during pregnancy has in the past been incorrectly interpreted as indicative of increased thyroidal hormone production and activity. Although the basal metabolic rate increases progressively throughout pregnancy by up to $25 \%$, this increase is almost exclusively due to the fetoplacental unit and increased work of the maternal heart (Burrow, 1975). The increase in protein bound iodine (PBI), serum thyroxine $\left(T_{4}\right)$ to a lesser extent $T_{3}$ and biologically inactive reverse $T_{3}$ is principally due to an increase in circulating thyroid hormone-binding proteins, in particular thyroxine binding globulin (TBG). There is a doubling of TBG during the first trimester due to oestrogen-stimulated hepatic production. Thus the absence of a TBG and $T_{4}$ rise early in pregnancy may indicate poor oestrogen production and possible fetal loss. The increase in serum $T_{4}$ and $T_{3}$ is maximal during the second trimester (Chan et al., 1975) and these hormones return to normal levels during the puerperium.

Thyroid hormones are highly $(>99 \%)$ protein bound and it is the free unbound fraction that exerts biological activity. There is general agreement (Innerfield and Hollander, 1977) that free hormone concentration is not increased during pregnancy. However, the routine assay of free hormones is complex and has not to date been widely available. The free thyroxine index (FTI), derived from the measurement of $T_{4}$ and binding capacity and the $T_{3}$ uptake, $\left(T_{4} / T_{3}\right.$ uptake $\left.\times 100=F T I\right)$ gives an indirect approximation of the free hormone concentration, which compensates for the alteration in thyroid hormone-binding proteins. This remains unchanged during pregnancy and is at present the single most reliable routine test of thyroid function. The 'effective thyroxine ratio' gives similar results in pregnancy.

Thyroxine turnover during pregnancy has been studied following the injection of ${ }^{13}$ I-labelled $T_{4}$ (Dowling et al., 1967). As a result of a decrease in the proportion of free $T_{4}$, the volume of distribution and fractional turnover are decreased slightly but, following correction for the change in surface area, the net thyroxine turnover and hormonal requirements are unchanged by pregnancy. Serum TSH levels and the response of TSH to thyrotrophinreleasing hormones (TRH) have been reported both as unaltered (Kanazawa et al., 1976) and increased during pregnancy (Burrow, 1978). The suggestion of enhanced responsiveness of TSH to TRH is also evidence against increased thyroid activity as a minimal rise in circulating thyroid hormone usually decreases responsiveness to TRH.

\section{Placental thyroid stimulators}

Three glycoprotein thyroid stimulators have been identified in the normal placenta, namely chorionic gonadotrophin (HCG), human chorionic thyrotrophin (HCT) and human molar thyrotrophin (HMT, the last so called because it is produced in large amounts by hydatidiform moles and choriocarcinoma). HCT is similar in molecular size to TSH and has a short duration of action (peak $\sim 3 \mathrm{hr}$ ). Its content in normal placenta is usually small, of the order of $10 \mathrm{mu}$. per placenta (Kenimer, Hershman and Higgins, 1975). HMT, on the other hand, is of larger molecular size with a longer duration of action and it has been suggested that it may act as a precursor for HCT (Tunbridge and Hall, 1975).

HCG is, in addition, a weak thyroid stimulator, on a molecular basis containing $1 / 4000$ the thyrotrophic activity of pituitary TSH, and there is some evidence that HMT may in fact be HCG (Kenimer et al., 1975). In normal pregnancy there is probably sufficient HCG produced to stimulate the maternal thyroid gland during the first trimester. HCT is also present from early pregnancy but unlike HCG its concentration rises progressively throughout pregnancy (Kanazawa et al., 1976), which would lead to progressive thyroidal enlargement if it were the placental thyrotrophin.

\section{Fetal thyroid function}

The fetal hypothalamic-pituitary-thyroidal system develops and functions autonomously. The trans placental passage of $T_{4}$ and $T_{3}$ is minimal both in animals and man (Fisher et al., 1977). There is no: correlation between maternal and fetal concentrations of $\mathrm{T}_{4}, \mathrm{~T}_{3}$ or TSH at any time during gestation despite a concentration gradient. Furthermore, only minimal proportions of $T_{4}$, or radioiodine-labelled $T_{4}$, given to mothers before labour or therapeutic abortion have been detected in the fetus. Animal studies support this conclusion, but biologically active thyroid hormone analogues may cross the placenta in some species (Burrow, 1978).

The fetal thyroid does not secrete thyroid hormone until the end of the first trimester and its development proceeds in the absence of TSH. There is an abrupt rise in fetal TSH concentrations between 18 and 24 weeks correlated temporarily with histological maturation of the hypothalmic-pituitary portal vascular system, which results in a marked increase in thyroidal production of $T_{4}$ and $T_{3}$. The concentration of $T_{4}$, especially free $T_{4}$ (as fetal TBG concentration does not increase), rises slowly after 30 weeks to that of the mother at term, whereas the elevated fetal TSH levels decline somewhat towards term (Fisher et al., 1977).

The peripheral de-iodination of $\mathrm{T}_{4}$ is the major source of production of $T_{3}$ and almost exclusively the source of reverse $T_{3}$. In the fetus, the peripheral de-iodination of thyroxine favours the production of biologically inactive reverse $T_{3}$ at the expense of 
active $T_{3}$; thus cord blood $T_{3}$ concentration is approximately one fifth that of maternal (Chopra, Sack and Fisher, 1975).

During the neonatal period there is a marked increase in serum TSH, in part a response to neonatal cooling, reaching a peak at $30 \mathrm{~min}$. Serum $\mathrm{T}_{3}$ also increases, reaching an early peak at $2 \mathrm{hr}$ and a second peak coincidental with the $T_{4}$ and free $T_{4}$ peak at $24 \mathrm{hr}$. This period of neonatal thyroid hyperactivity is transient, falling gradually over 2 to 3 days for TSH and 2 to 3 weeks for the thyroid hormones. Reverse $T_{3}$ levels do not peak and return to adult range within 10 to 14 days suggesting maturation of the peripheral enzymatic pathway of thyroxine metabolism. It is now possible to recommend screening of the newborn for hypothyroidism, based on $\mathrm{T}_{4}$ or TSH, or preferably both, on either cord blood or heel prick 3 to 5 days post partum (Dussault et al., 1975). The incidence of neonatal hypothyroidism varies from $1 / 4000$ in a number of European countries to $1 / 7000$ in Quebec.

\section{Acknowledgments}

I wish to thank Professor J. Crooks for his help in the preparation of this paper.

\section{References}

Aboul-Khair, S.A., Crooks, J., Turnbull, A.C. \& Hytten, F.E. (1964) The physiological changes in thyroid function during pregnancy. Clinical Science, 27, 195.

Burrow, G.N. (1975) The thyroid in pregnancy. Medical Clinics of North America, 59 No (5), 1089.

BURROW, G.N. (1978) Hyperthyroidism during pregnancy. New England Journal of Medicine, 298, 150.

Chan, V., Paraskevaides, C.A. \& Hale, J.F. (1975) Assess- ment of thyroid function during pregnancy. British Journal of Obstetrics and Gynaecology, 82, 137.

ChOPRA, I.J., SACK, J. \& Fisher, D.A. (1975) Circulating $3,3^{\prime}, 5^{\prime}$-triiodothyronine (reverse $T_{3}$ ) in the human newborn. Journal of Clinical Investigation, 55, 1137.

Crooks, J., Aboul-Khair, S.A., Turnbull, A.C. \& Hytten, F.E. (1964) The incidence of goitre during pregnancy. Lancet, ii, 334.

Crooks, J., Tulloch, M.I., Turnbull, A.C., Davidson, D., Skulason, T. \& Snaedal, G. (1967) Comparative incidence of goitre in pregnancy in Iceland and Scotland. Lancet, ii, 625.

Dowling, J.T., Appleton, W.G. \& Nicoloff, J.T. (1967) Thyroxine turnover during human pregnancy. Journal of Clinical Endocrinology and Metabolism, 27, 1749.

Dussault, J.H., Coulombe, P., Laberge, C., Lelarle, J., Guyda, H. \& Khoury, K. (1975) Preliminary report on mass screening program for neonatal hypothyroidism. Journal of Pediatrics, 86, 670.

Fisher, D.A., Dussault, J.H., SACK, J. \& Chopra, I.J. (1977) Ontogenesis of hypothalamic-pituitary-thyroid function and metabolism in man, sheep and rat. Recent Progress in Hormone Research, 33, 59.

Galina, M.P., Avnet, N.L. \& Einhorn, A. (1962) Iodides during pregnancy - an apparent cause of neonatal death. New England Journal of Medicine, 267, 1124.

INNERFIELD, R. \& HOLLANDER, C.S. (1977) Thyroidal complications of pregnancy. Medical Clinics of North America, 61 (1), 67.

Kanazawa, S., Nakamura, A., Saida, K. \& Tojo, S. (1976) Placento-thyroidal relationship in normal pregnancy. Acta obstetrica et gynecologica scandinavica, 55, 201.

Kenimer, J.G., Hershman, J.M. \& Higgins, H.P. (1975) The thyrotrophin in hydatidiform moles is human chorionic gonadotrophin. Journal of Clinical Endocrinology and Metabolism, 40, 482.

Malkasian, G.D. \& MaYberRy, W.E. (1970) Serum total and free thyroxine and thyrotrophin in normal and pregnant women, neonates, and women receiving progestogens. American Journal of Obstetrics and Gynecology, 108, 1234.

TUNBRIDGE, W.M.G. \& HALL, R. (1975) Thyroid function in pregnancy. Clinics in Obstetrics and Gynecology. 2, (2), 381. 